\title{
Molecular mechanisms of the antimetastatic activity of nuclear clusterin in prostate cancer cells
}

\author{
ROBERTA M. MORETTI ${ }^{1}$, STEFANIA MAI ${ }^{1}$, MARINA MONTAGNANI MARELLI ${ }^{1}$, \\ FEDERICA RIZZI $^{2}$, SAVERIO BETTUZZI ${ }^{2}$ and PATRIZIA LIMONTA ${ }^{1}$ \\ ${ }^{1}$ Department of Endocrinologia, Fisiopatologia e Biologia Applicata, Università degli Studi di Milano, \\ I-20133 Milan; ${ }^{2}$ Department of Medicina Sperimentale, Università degli Studi di Parma, \\ I-43100 Parma; ${ }^{3}$ INBB, Istituto Nazionale Biostrutture e Biosistemi, I-00136 Rome, Italy
}

Received January 10, 2011; Accepted February 16, 2011

DOI: 10.3892/ijo.2011.1030

\begin{abstract}
The proapoptotic activity of nuclear clusterin (nCLU) in cancer cells is now well established. We previously showed that nCLU decreases the motility of prostate cancer cells by triggering a dramatic dismantling of the actin cytoskeleton. Here, we sought to unravel the molecular mechanisms of the antimetastatic activity of nCLU. We found that nCLU: i) decreases LIMK1 expression, thus increasing the levels of the active (unphosphorylated) form of cofilin, the well known actin depolymerizing factor; ii) binds to vimentin, sequestering the protein from its adhesion sites at the cell periphery, thus interfering with its role in cell motility and adhesion; iii) affects the intracellular distribution of E-cadherin (the major component of epithelial adherens junctions) which appears to be diffusely distributed in the cells. Through these mechanisms nCLU reduces the migratory/invasive behavior of PC3 cells; this effect is further demonstrated by a decreased secretion of active MMP-2 from the cells. Thus, in addition to its proapoptotic function, nCLU also exerts a strong anti-migratory/anti-invasive activity in prostate cancer cells, by interfering with the cytoskeletal components and by decreasing MMP-2 activity.
\end{abstract}

\section{Introduction}

Prostate cancer (PCa) still remains a significant medical burden and a major cause of cancer-related death in Western countries (1). Most PCas are dependent on the presence of androgens for growth and survival; thus, for advanced androgen-dependent $\mathrm{PCa}$, androgen ablation therapy represents the most effective initial treatment (2). Unfortunately, despite castrate serum androgen levels, most $\mathrm{PCa}$ patients develop disease progression

Correspondence to: Dr Patrizia Limonta, Department of Endocrinologia, Fisiopatologia e Biologia Applicata, Università degli Studi di Milano, Via Balzaretti 9, I-20133 Milan, Italy

E-mail: patrizia.limonta@unimi.it

Key words: nuclear clusterin, cell motility, cell invasion, prostate cancer, cytoskeleton towards androgen-independent or castration resistant stage, characterized by high proliferation rate, invasion and malignancy (3). Once this stage of hormone-refractoriness is reached, conventional chemotherapy treatments have provided scant benefits (4). In recent years, several new agents, focused on improved chemotherapeutics or on different targets such as angiogenesis and immune system, have been developed and are at present under investigation $(5,6)$. A better understanding of the molecular mechanisms underlying the development of malignant behavior in androgen-independent $\mathrm{PCa}$ will help increase the therapeutic options for this almost uncurable neoplasia.

Clusterin (CLU), expressed in several tissues and fluids, has been shown to be involved in different biological processes, including carcinogenesis and tumor progression $(7,8)$. Since its first discovery, many studies have been performed to clarify the structure of this fascinating protein. So far, two main isoforms of CLU have been identified in humans, both generated by the translation of a single 9-exon gene located on chromosome 8 (9). The most studied and best known isoform is a glycosylated heterodimeric protein whose translation starts from the AUG codon located in the second exon. The protein precursor is driven by a signal peptide to the endoplasmic reticulum, where it is cleaved into two distinct peptides ( $\alpha$ and $\beta$ ) held together by five disulphide bridges, then transported to the Golgi, where it undergoes heavy glycosylation before being secreted. This isoform is named secreted CLU (sCLU) $(10,11)$. The second isoform is a $49-\mathrm{kDa}$ protein, usually induced by proapoptotic stimuli and targeted to the nucleus. For this reason it is named nuclear CLU (nCLU) $(11,12)$. We found that a similar protein $(49 \mathrm{kDa})$ can be produced when synthesized from a second AUG codon (position 152) (13). This protein is unglycosylated, lacks the signal peptide and appears to localize mainly at the nuclear level. The molecular mechanisms underlying the generation of the two CLU isoforms (alternative splicing/alternative initiation of translation?) are still a matter of intensive debate $(14,15)$.

The two CLU isoforms have been reported to play distinct roles in the control of cell growth processes in different types of cancer. sCLU, a stress-activated molecular chaperone, has been shown to be overexpressed in many tumors (16-20). It functions as a survival factor protecting cells from many 
therapeutic stressors that induce apoptosis, such as radiation, steroid-withdrawal, and chemotherapy (21-24). On the other hand, nCLU has been reported to accumulate in the nucleus of cells challanged with different apoptotic stimuli $(15,25)$. Thus, it seems that sCLU behaves as a prosurvival factor, whereas nCLU is proapoptotic.

In prostate cancer, the specific role of the two CLU isoforms in tumor development and progression is still a controversial issue (26). CLU expression has been reported to be either up(27) or down-regulated (28-30) in different models of PCa. High serum levels of sCLU have been detected in PCa patients $(31,32)$. In PCa cells, sCLU has been shown either to favor cell survival and to mediate resistance to treatment-induced apoptosis (33-37) or to inhibit cell proliferation $(26,38)$. On the other hand, nCLU has been consistently demonstrated to mediate the activity of different proapoptotic signals $(30,38,39)$. Thus, while the effects of sCLU on prostate cancer growth are still a matter of debate, the proapoptotic function of nCLU is generally accepted.

In a previous work (40), we reported that nCLU can be produced in SV40-immortalized epithelial prostate cells PNT1A starting form an expression vector carrying the full length cDNA for human CLU, while the nuclear form is hardly produced in androgen-independent, metastatic PC3 cells. We succeeded at expressing high levels of nCLU in PC3 cells using an expression vector in which a truncated form of human CLU, lacking the leader peptide, was cloned. In this system, the two protein isoforms differentially affected the growth of androgen-independent PC3 cells, with sCLU being ineffective and nCLU exerting a strong antiproliferative activity. We concluded that sCLU (cytoprotective) and nCLU (proapoptotic) isoforms are both synthesized in immortalized benign PNT1A prostate epithelial cells, while metastatic cancer PC3 cells escape from the proapoptotic activity of CLU by blocking nCLU production (40). This suggests that a shift in the production of the two isoforms might play a crucial role in prostate tumorigenesis (40). Interestingly, in the same work, we also found that nCLU, but not sCLU, is endowed with a strong antimotility activity in PC3 cells (40). On the basis of these results, we came back to the same experimental system, to get further insights into the molecular mechanisms underlying the antimetastatic properties of nCLU. To do so, we have investigated possible changes in expression and intracellular localization of several key proteins of the cytoskeleton when nCLU is expressed in PC3 cells. The results shown confirm the peculiar antitumor activity of nCLU, strongly supporting the idea that shifting the balance between sCLU and nCLU (i.e., increasing nCLU while decreasing sCLU) might represent a potential new treatment strategy for androgen-independent PCa.

\section{Materials and methods}

Materials. Antibodies used: mouse anti-human clusterin antibody (clone 41D) from Upstate Biotechnology (Lake Placid, NY); rabbit anti-human cofilin antibody, rabbit antihuman phospho-cofilin (Ser3) antibody, and rabbit anti-human LIMK1 antibody from Cell Signaling Technology, Beverly, MA; mouse anti-human $\beta$-tubulin (clone TUB 2.1), and goat anti-human vimentin from Sigma Chemical Co. (St. Louis,
MO); goat anti-human E-cadherin from R\&D Systems, Inc. (Minneapolis, MN); goat anti-human actin (SC-1616) from Santa Cruz Biotechnology (Santa Cruz, CA); FITC-conjugated goat anti-mouse secondary antibody (Alexa Fluor 488), TRITCconjugated goat anti-mouse secondary antibody (Alexa Fluor 594), FITC-conjugated rabbit anti-goat secondary antibody (Alexa Fluor 488), and TRIC-conjugated rabbit anti-goat secondary antibody (Alexa Fluor 594) from Molecular Probes Inc. (Eugene, OR); secondary horseradish-peroxidaseconjugated rabbit anti-mouse antibody from Sigma Chemical Co.; secondary horseradish-peroxidase-conjugated rabbit anti-goat antibody from Santa Cruz Biotechnology.

Cell cultures. The human androgen-independent PC3 prostate cancer cell line was purchased from the American Tissue Culture Collection (Manassas, VA, USA). Cells were routinely grown in Roswell Memorial Institute-1640 (RPMI-1640) medium supplemented with $10 \%$ fetal bovine serum (FBS), glutamine $(1 \mathrm{mmol} / \mathrm{l})$ and antibiotics $(100 \mathrm{IU} / \mathrm{ml}$ penicillin $\mathrm{G}$ sodium and $100 \mu \mathrm{g} / \mathrm{ml}$ streptomycin sulfate), in a humidified atmosphere of $5 \% \mathrm{CO}_{2} / 95 \%$ air at $37^{\circ} \mathrm{C}$.

Expression vectors and transfections. Human nuclear clusterin (nCLU) cDNA was generated as described (38). Briefly, a truncated CLU cDNA fragment was amplified from the 'fulllength' CLU cDNA using the following primers: 5'-GTC TCA GAC AAT GGG ATC CAG GA-3' (forward); and 5'-GAC CTG CAG GCG GCC GCG AAT-3' (reverse). Truncated cDNA was inserted in pIRES-hyg to generate pIRES-nCLU vector (38). Constructs were sequenced before carrying out expression experiments. Transfections were performed with the nonliposomal reagent FuGENE 6 (Roche, Mannheim, Germany). Cells $\left(10^{5}\right)$ were plated in $35-\mathrm{mm}$ dishes and transiently transfected with $3 \mu \mathrm{g} / \mathrm{dish}$ of plasmid DNA (pIRES-nCLU) for different time intervals $(24,48,72,120 \mathrm{~h})$. In each experiment, control cells were transfected with the empty vector pIRES-hyg. Efficiency of transfection was routinely assessed by green fluorescence protein (GFP) expression using the pCMVGFP-LpA vector (Clontech Laboratories, Palo Alto, CA). Transfection efficiency was usually higher than $40 \%$ of total cells. The size of the pCMV-GFP-LpA vector is comparable with that of the vector used for CLU overexpression. High efficiency of the procedure used is demonstrated by the detection of the protein bands by Western blotting (Fig. 1).

Western blot analysis. Transfected cells for $48 \mathrm{~h}$ were lysed in RIPA buffer (0.05 M Tris-HCl pH 7.7, $0.15 \mathrm{M} \mathrm{NaCl,} 0.8 \%$ SDS, $10 \mathrm{mM}$ EDTA, $100 \mu \mathrm{M} \mathrm{NaVO}_{4}, 50 \mathrm{mmol} / \mathrm{l} \mathrm{NaF}, 0.3 \mathrm{mM}$ PMSF, $5 \mathrm{mM}$ iodoacetic acid) containing leupeptin $(50 \mu \mathrm{g} / \mathrm{ml})$, aprotinin $(5 \mu \mathrm{l} / \mathrm{ml})$ and pepstatin $(50 \mu \mathrm{g} / \mathrm{ml})$. The extracts were centrifuged to remove insoluble material. Protein concentration was determined using the BCA method. Protein extracts $(30 \mu \mathrm{g})$ were resuspended in sample buffer $(0.5 \mathrm{M}$ Tris- $\mathrm{HCl} \mathrm{pH} 6.8$, $20 \%$ glycerol, $10 \%$ SDS, $0.2 \% 2 \beta$-mercaptoethanol, $0.05 \%$ blue bromophenol) and heated at $95^{\circ} \mathrm{C}$ for $5 \mathrm{~min}$. Following electrophoretic separation by $7.5 \%$ SDS-PAGE for E-cadherin, $10 \%$ SDS-PAGE for clusterin, LIMK-1, $\beta$-tubulin and vimentin, and $12 \%$ SDS-PAGE for cofilin and p-cofilin, proteins were transferred onto nitrocellulose membranes. Membranes were blocked in 3\% BSA prior to incubation at room temperature 
for $2 \mathrm{~h}$ with the primary antibody (1:1000). Detection was done using a horseradish-peroxidase-conjugated secondary antibody and enhanced chemiluminescence reagents (Supersignal Chemiluminescence Detection System, Pierce Biotechnology Inc., Rockford, IL). Actin expression was evaluated as a loading control, since we previously showed that nCLU overexpression does not affect actin protein expression (40). Protein preparations $(30 \mu \mathrm{g})$ were processed for Western blotting as described above, with goat anti-human (1:2000) as the primary antibody.

Co-immunoprecipitation. Transfected PC3 cells (48 h) were lysed in RIPA buffer. Extracts were centrifuged to pellet the cell debris. Protein extracts from supernatants were incubated with anti-clusterin antibody $(2 \mu \mathrm{g})$ and rabbit anti-mouse immunoglobulin $\mathrm{G}$ at room temperature for $2 \mathrm{~h}$. Protein A-Sepharose beads were added and incubated overnight at $4{ }^{\circ} \mathrm{C}$ with gentle rotation. After centrifugation, immunoprecipitated pellets were washed five times with ice-cold wash RIPA buffer and thrice with water in refrigerated microcentrifuge. The pellets were dissolved in reducing sample buffer, electrophoresed and blotted onto nitrocellulose membranes. Membranes were blocked with $3 \%$ BSA and incubated $2 \mathrm{~h}$ at room temperature with mouse anti-human $\beta$-tubulin $(1: 1000)$ or with goat antihuman vimentin (1:500). Membranes were then incubated with rabbit anti-mouse or rabbit anti-goat secondary antibody, respectively, for $1 \mathrm{~h}$ at room temperature. Signal was detected with enhanced chemiluminescence reagents (Super Signal Chemiluminescence Detection System).

Immunofluorescence analysis. PC3 cells were seeded on 13-mm diameter coverslips. After transfection (48 h), cells were fixed with $3 \%$ paraformaldehyde in $2 \%$ sucrose-PBS for $15 \mathrm{~min}$ and permeabilized with $0.5 \% \mathrm{HEPES} /$ Triton buffer (20 mM HEPES, $300 \mathrm{mM}$ sucrose, $50 \mathrm{mM} \mathrm{NaCl}, 3 \mathrm{mM}$ $\mathrm{MgCl}_{2}, 0.5 \%$ Triton X-100) for $1 \mathrm{~min}$.

Detection of $\beta$-tubulin and vimentin: cells were stained with the unlabeled monoclonal primary antibodies at the appropriate dilutions (1:200 for anti- $\beta$-tubulin; 1:20 for antivimentin) followed by FITC-conjugated goat anti-mouse secondary antibody (Alexa Fluor 488).

Detection of E-cadherin: cells were stained with the unlabeled monoclonal anti-E-cadherin primary antibody (1:20) followed by TRITC-conjugated rabbit anti-goat secondary antibody (Alexa Fluor 594).

Analysis of nCLU/vimentin co-localization: nCLU-transfected PC3 cells were fixed and incubated with the unlabeled monoclonal anti-clusterin primary antibody (1:100) followed by TRITC-conjugated goat anti-mouse secondary antibody (Alexa Fluor 594). Cells were then incubated with the goat anti-human vimentin primary antibody (1:20), followed by FITC-conjugated rabbit anti-goat secondary antibody (Alexa Fluor 488).

Analysis of nCLU/E-cadherin co-localization: nCLUtransfected PC3 cells were fixed and incubated with the unlabeled monoclonal anti-clusterin primary antibody (1:100) followed by FITC-conjugated goat anti-mouse secondary antibody (Alexa Fluor 488). Cells were then incubated with the goat anti-human E-cadherin primary antibody (1:20), followed by TRITC-conjugated rabbit anti-goat secondary antibody (Alexa Fluor 594).
In each experiment, labeled cells were examined under a Zeiss Axiovert 200 microscope with a 63x/1.4 objective lens linked to a Coolsnap Es CCD camera (Roper Scientific-Crisel Instruments, Rome, Italy).

Invasion assays. The invasiveness of PC3 cells was assayed using modified transwell Boyden's chambers (Neuro Probe Inc., Gaithersburg, MD). Polycarbonate filters (pore size, $8 \mu \mathrm{m}$ ) separating the upper and lower compartments were coated with a thin layer of ECM-Matrigel $\left(100 \mu \mathrm{g} / \mathrm{cm}^{2}\right)(\mathrm{BD}$ Biosciences, San Jose, CA) for $1 \mathrm{~h}$ at $37^{\circ} \mathrm{C}$. Transfected cells $(48 \mathrm{~h})$ were counted and placed in the upper compartment of a Boyden's chamber $\left(10^{5}\right.$ cells $\left./ 50 \mu \mathrm{l}\right)$. The lower compartment of the chamber filled with serum free medium containing laminin $(20 \mu \mathrm{g} / \mathrm{ml})$, as the chemoattractant. After $24 \mathrm{~h}$ of incubation at $37^{\circ} \mathrm{C}$, cells that had invaded the Matrigel were fixed, stained (Diff-Quick kit, DADE, Dudingen, Switzerland) and mounted onto glass slides. Six random objective fields of stained cells were counted for each well (12 wells/experimental group) and the mean number of invading cells $/ \mathrm{mm}^{2}$ was calculated. Assays were repeated three times. Data were analyzed according to the Bonferroni's test after One-way ANOVA.

Zymographic analysis. PC3 cells were seeded in flasks at a density of $10^{6}$ cells/flask in serum-free medium for 2 days. After 2 days cells were plated in 35-mm dishes and transiently transfected in serum free medium for $48 \mathrm{~h}$. At the end of the transfection, the conditioned media were collected, centrifuged at $12000 \mathrm{rpm}$ for $10 \mathrm{~min}$ at $4^{\circ} \mathrm{C}$ and resuspended in non-reducing sample buffer. Samples were separated on $7.5 \%$ polyacrylamide gels containing $0.5 \mathrm{mg} / \mathrm{ml}$ gelatin. After electrophoresis, gels were washed three times in $2.5 \%$ Triton X-100 for 10 min two times in substrate buffer (1X) containing $50 \mathrm{mM}$ Tris- $\mathrm{HCl}$ (pH 7.5), $5 \mathrm{mM} \mathrm{CaCl}_{2}, 200 \mathrm{mM} \mathrm{NaCl}, 0.02 \%$ Brij-35 (Bio-Rad Laboratories, Segrate, Italy), and incubated in the same buffer at $37^{\circ} \mathrm{C}$ overnight. Gels were stained for $60 \mathrm{~min}$ in $40 \%$ methanol/ $10 \%$ glacial acetic acid containing $0.1 \%$ Coomassie brillant blue. MMP-2 activity was visualized in the gelatin-containing zymograms as clear bands against a blue background.

Statistical analysis. When appropriate, data were analyzed by Bonferroni's test, after one-way analysis of variance.

\section{Results}

Effects of $n C L U$ overexpression in metastatic PC3 cells: efficiency of transfection, dismantling of the cytoskeleton, inhibition of migration. In a previous report (40) we showed that nCLU overexpression decreases the motility of androgenindependent PC3 prostate cancer cells by dismantling the actin cytoskeleton. To unravel the mechanisms underlying the antimotility activity of nCLU, and to clarify whether it may also affect the invasive behavior of PCa cells, we efficiently expressed nCLU in the PC3 cell system as previously reported (40). Our experiments confirmed the efficiency of the cell transfection system. Fig. 1 shows that a $49-\mathrm{kDa}$ protein band, corresponding to the molecular weight of nCLU isoform, unglycosylated and lacking the leader peptide, is effectively expressed in PC3 cells from 24 up to $120 \mathrm{~h}$ after transfection with the pIRES-nCLU plasmid. Experiments were then 


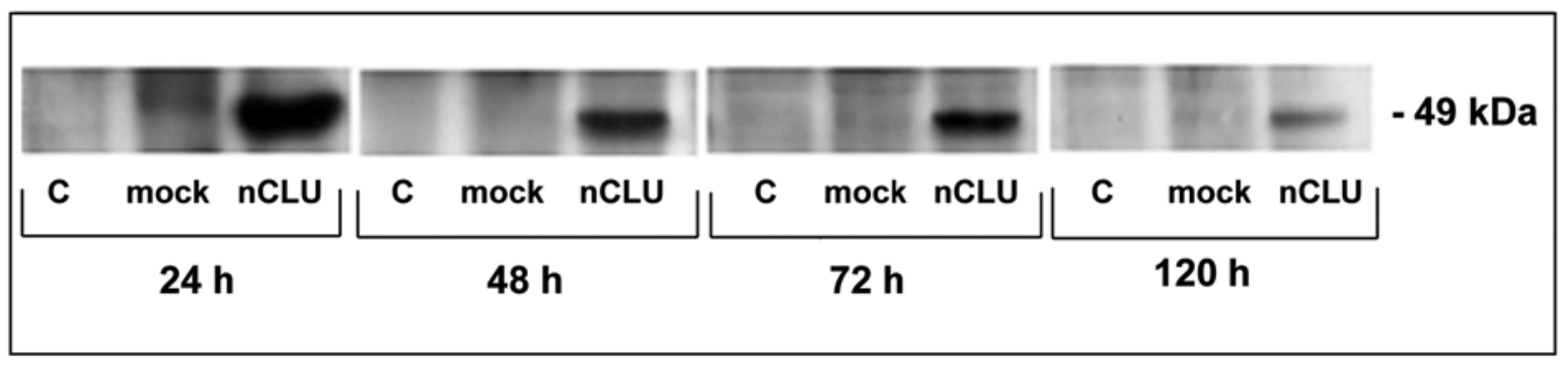

Figure 1. Overexpression of nCLU in PC3 prostate cancer cells. Western blot analysis of nCLU in whole-cell extracts of transiently (24-120 h) transfected PC 3 cells. Representative of two different experiments with similar results.

A

B

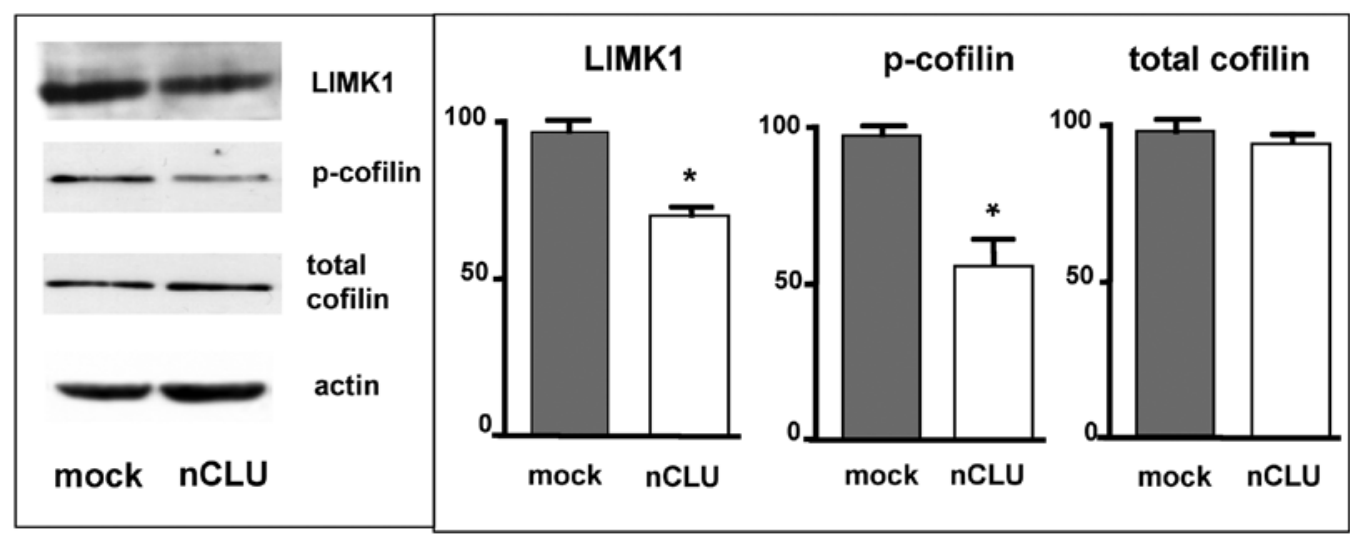

Figure 2. nCLU overexpression affects LIMK1 and p-cofilin expression in PC3 cells. (A) Western blot analysis of LIMK1, p-cofilin, and total cofilin in whole-cell extracts of transiently $(48 \mathrm{~h})$ transfected PC 3 cells. Actin expression is used as a loading control. Representative pictures from three experiments done independently, which gave the same results. (B) Densitometric analysis of the data showed in (A). The diagram shows a quantification of three assays. Mock, mock-transfected cells; nCLU, nCLU-transfected cells. "P<0.05 vs. mock.

perfomed at $48 \mathrm{~h}$ after transfection. Under these conditions, we also confirmed that the actin cytoskeleton is completely dismantled and the migratory capacity of PC 3 cells is significantly inhibited, as previously observed (40). It is important to underline that the antimotility effect of nCLU can not be influenced by a decreased number of nCLUoverexpressing cells. Actually, cell death induction by nCLU is a process which takes from $24 \mathrm{~h}$ to a few days to be fully appreciated in prostate cells (41); the migratory test performed in our laboratory takes only $4 \mathrm{~h}$.

$n C L U$ reduces LIMK1 expression, thus increasing the amount of unphosphorylated, active form of cofilin (actin-depolimerizing factor). We previously showed that nCLU binds to $\alpha$-actinin, a key protein in the actin cytoskeleton organization, and hypothesized that this mechanism might be responsible, at least partially, for the dramatic dismantling of the actin cytoskeleton induced by the CLU isoform. Here, we focused our attention on cofilin (also known as actin-depolimerizing factor, ADF), another actin-binding protein, considered to be a potent regulator of the actin dynamics, by means of its ability to induce F-actin depolymerization (42). Cofilin is active in its unphosphorylated form; it is actually well known that the phosphorylation of cofilin at the Ser-3 residue, by means of the LIMK1 kinase, abrogates its activity on actin organization
(43). By Western blotting, we showed that, in PC3 cells transfected with nCLU, the expression of LIMK1 was significantly decreased (Fig. 2). As expected, the amounts of the phosphorylated form of cofilin (p-cofilin) were also reduced by nCLU, while total cofilin levels (phosphorylated plus unphosphorylated form) were unchanged, pointing to an increase of the unphosphorylated, active, form of the protein (Fig. 2). These data indicate that the dramatic dismantling effect of nCLU on the actin cytoskeleton is mediated not only by its binding to $\alpha$-actinin, but also by its ability to activate cofilin, the actin depolymerizing factor, through a decreased expression of the LIMK1.

$n C L U$ does not affect microtubule organization. Microtubules are the main cytoskeletal component involved in the formation and disappearance of the mitotic spindle, which is responsible for cell division. For this reason, microtubule-stabilizing and microtubule-destabilizing agents are commonly used as anticancer agents (44). However, microtubules are also well known for playing a crucial role in cell polarization and directional cell migration (45). On the basis of this observation, we sought to investigate whether nCLU overexpression might affect microtubule organization in prostate cancer cells. By immunofluorescence, we found that the microtubule network is well organized in both control and mock-transfected PC3 
A

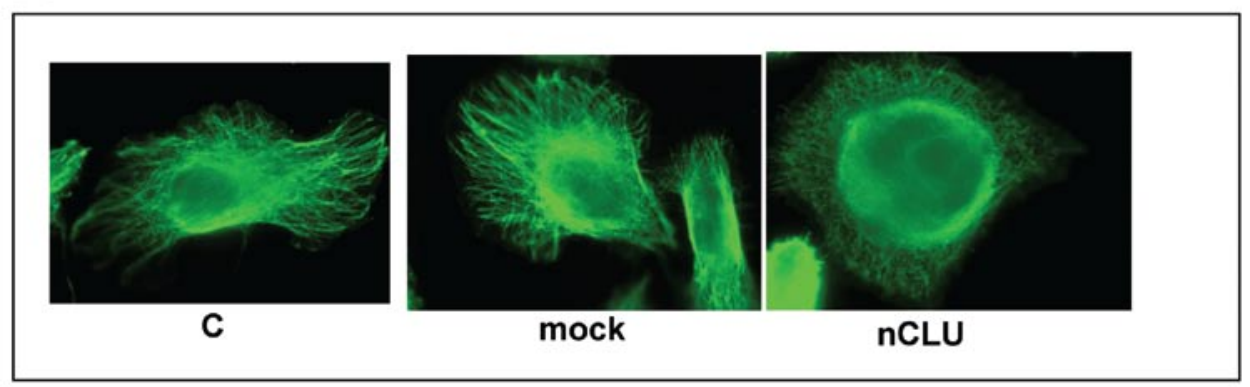

B

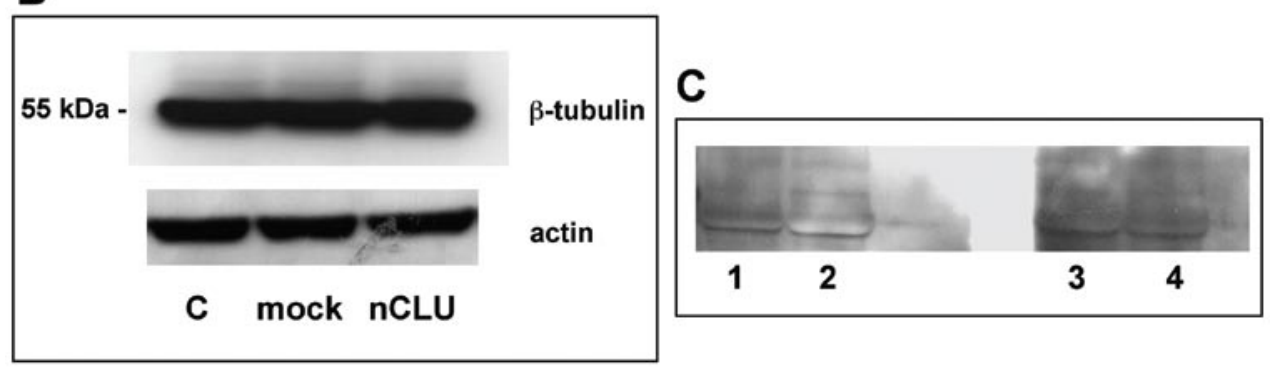

Figure 3. nCLU overexpression does not affect microtubule organization in PC3 cells. (A) Immunofluorescence staining of $\beta$-tubulin in untransfected control (C), mock-transfected (mock), and nCLU-transfected (nCLU) PC3 cells $48 \mathrm{~h}$ after transfection. Images were taken at magnification x630. (B) Western blot analysis of $\beta$-tubulin in whole-cell extracts of transiently ( $48 \mathrm{~h})$ transfected PC 3 cells. Actin expression is used as a loading control. (C) Co-immunoprecipitation experiment on PC3 cells transiently $(48 \mathrm{~h})$ transfected with nCLU. Protein extracts from PC3 cells were immunoprecipitated with anti-clusterin antibody, resolved by SDS-PAGE, and processed for immunoblotting with anti- $\beta$-tubulin antibody (lane 2 ). Lane 1 , protein extracts from mock-transfected cells; lane 3 , no cell extracts with anti-clusterin antibody; lane 4, protein extracts from transfected cells without anti-clusterin antibody. No co-immunoprecipitation bands can be detected in any experimental sample. Representative pictures from three experiments done independently, which gave the same results.

cells (Fig. 3A); moreover, nCLU overexpression does not affect the microtubule assembly inside the cells (Fig. 3A). nCLU also did not modify $\beta$-tubulin expression in prostate cancer cells, when evaluated by Western blotting (Fig. 3B). Finally, coimmunoprecipitation experiments demonstrate that nCLU does not bind to $\beta$-tubulin (Fig. 3C, lane 2). No coimmunoprecipitation bands could also be detected in negative controls (Fig. 3C; lane 1, protein extracts from mocktransfected cells; lane 3, no cell extracts; lane 4, protein extracts without anti-clusterin antibody). Thus, the striking effects of nCLU on prostate cancer cell motility do not seem to be mediated by alterations of the cytoskeletal microtubule organization.

$n C L U$ binds to and co-localizes with vimentin. A body of evidence points to the intermediate filament vimentin as a promigratory molecule, which is upregulated during cell transformation (46). Specifically, vimentin is expressed in epithelial cells that undergo tumor invasion, and its level of expression has been correlated with motility of prostate cancer cells $(47,48)$. Here, we sought to investigate whether nCLU might affect the expression or the intracellular localization of vimentin. First, by immunofluorescence analysis, we showed that vimentin is highly expressed in control $(\mathrm{C}$, untransfected) as well as in mock-transfected (mock) PC3 cells (Fig. 4A), confirming previous observations (47). In these cells, vimentin is mainly localized at the cell periphery, accumulated at adhesion sites. After nCLU transfection, the intracellular localization of the intermediate filament is completely different, with vimentin being diffusely distributed into the cell (Fig. 4A,
nCLU). On the other hand, transfection of PC3 cells with nCLU does not affect the expression of vimentin, as evaluated by Western blotting (Fig. 4B). This indicates that nCLU overexpression significantly affects vimentin intracellular localization, without altering its expression level.

The 'chaperone'-like activity of clusterin has been previously demonstrated in different experimental models (7). Moreover, we previously reported that nCLU binds to $\alpha$-actinin, and this activity mediates, at least partially, the dramatic effects of nCLU on the actin cytoskeleton organization (40). Here, we sought to investigate whether nCLU might bind to the intermediate filament vimentin. To this purpose, co-immunoprecipitation analysis was done. Fig. 4C shows that, in PC3 cells overexpressing nCLU, the clusterin isoform specifically binds to vimentin (Fig. 4C, lane 2). No co-immunoprecipitation bands could be detected in negative controls (Fig. 4C; lane 1, protein extracts from mock-transfected cells; lane 3, no cell extracts; lane 4, protein extracts without anti-clusterin antibody). To further confirm this observation, colocalization analysis was done by immunofluorescence. Fig. 4D shows two cells (lanes 1 and 2) in which: nCLU is still present in the cytoplasm (lane 1) or is present both in the cytoplasm and in the nucleus (lane 2). These observations confirm our previous data showing that, after its overexpression, nCLU can localize not only at the nuclear but also at the cytoplasmic level (i.e., before entering the nucleus or, alternatively, behaving as a shuttle protein from the nucleus to the cytoplasm and vice versa) (40). Here we show that, in both cells, nCLU substantially colocalizes with vimentin, mainly in the perinuclear region (Fig. 4D, lanes 1 and 2, merge). Thus, nCLU binds to vimentin, possibly 
A

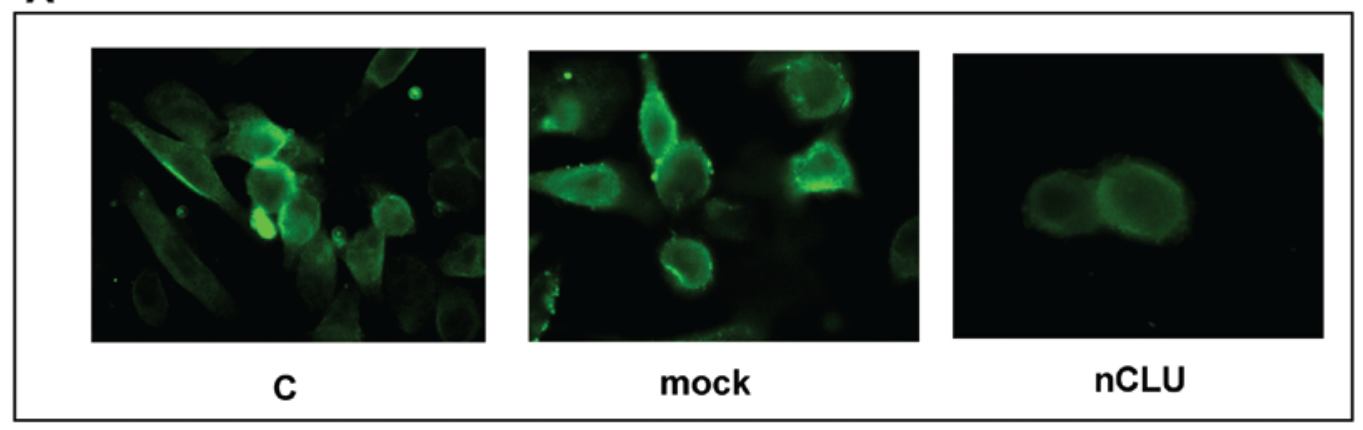

B

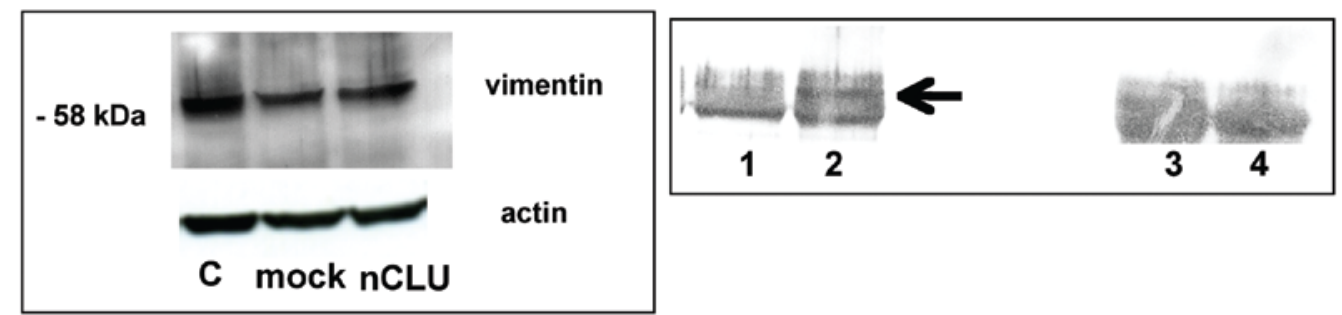

D

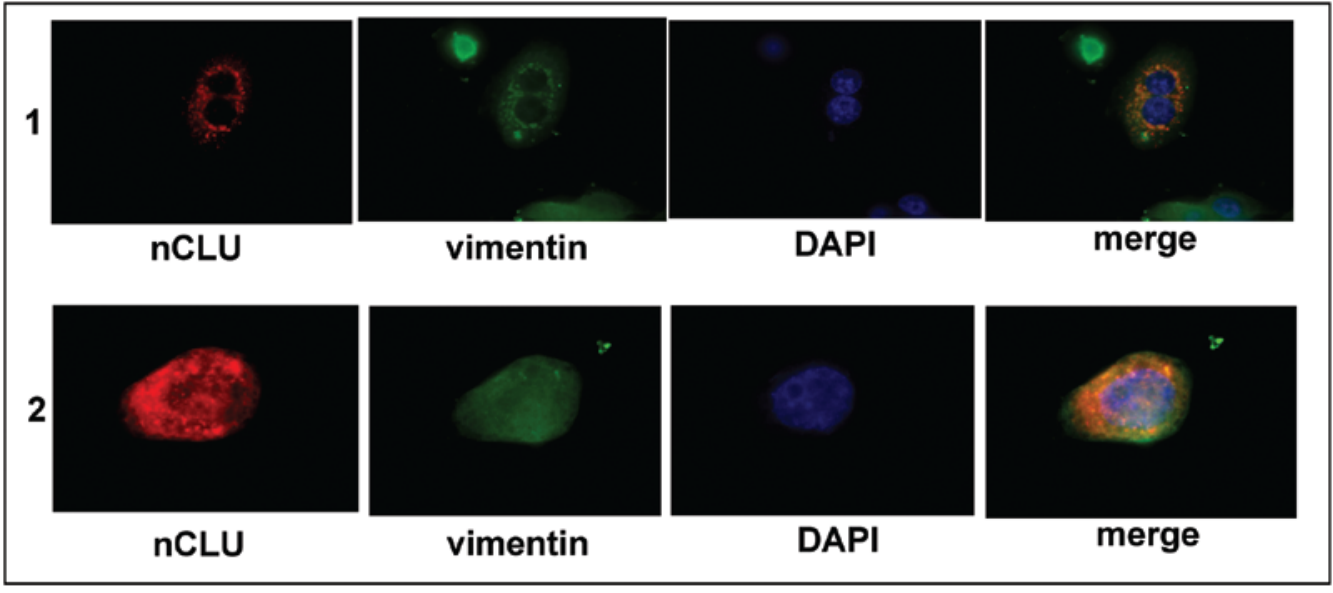

Figure 4. nCLU binds to and co-localizes with vimentin in transfected PC3 prostate cancer cells. (A) Immunofluorescence staining of vimentin in untransfected control (C), mock-transfected (mock), and nCLU-transfected (nCLU) PC3 cells $48 \mathrm{~h}$ after transfection. Images were taken under magnification x630. (B) Western blot analysis of vimentin in whole-cell extracts of transiently ( $48 \mathrm{~h}$ ) transfected PC 3 cells. Actin expression is used as a loading control Representative pictures from three experiments done independently, which gave the same results. (C) Co-immunoprecipitation experiment on PC3 cells transiently (48 h) transfected with nCLU. Protein extracts from PC3 cells were immunoprecipitated with anti-clusterin antibody, resolved by SDS-PAGE, and processed for immunoblotting with anti-vimentin antibody (lane 2). No co-immunoprecipitation band can be detected in negative controls: lane 1, protein extracts from mock-transfected cells; lane 3, no cell extracts with anti-clusterin antibody; lane 4, protein extracts from transfected cells without anti-clusterin antibody. Representative picture from three experiments done independently, which gave the same results. (D) Immunofluorescence staining of nCLU and vimentin in nCLU transfected PC3 cells. Lane 1, a transfected PC3 cell in which nCLU is still in the cytoplasm before entering the nucleus; lane 2, a transfected PC3 cell in which nCLU is present both in the cytoplasm and in the nucleus. In both cells, vimentin colocalizes with nCLU at the cytoplasmic level (merge). Images were taken at magnification x630. Representative pictures from three experiments done independently, which gave the same results.

sequestering it and interfering with its role in cell motility and adhesion.

nCLU modifies E-cadherin localization in prostate cancer cells. E-cadherin is the major component of epithelial adherens junctions. Modifications of E-cadherin expression/activity, and therefore of cell-cell contacts, have been widely shown to be involved in the motility of metastasizing cancer cells (49). Here, we investigated whether nCLU might affect E-cadherin expression and localization in PC3 cells. Fig. 5A shows that E-cadherin expression (by Western blotting) is not modified in prostate cancer cells overexpressing nCLU.
Immunofluorescence analysis shows that, in both control (C) and mock-transfected (mock) cells, E-cadherin is highly expressed and mainly localized at the cell periphery (cell-cell contacts) (Fig. 5B). Interestingly, most but not all of the cells (both controls and mock-transfected) express E-cadherin (Fig. 5B, C and mock), suggesting that PC3 cells represent a heterogeneous population, in which some cells do not express epithelial-related markers.

The intracellular distribution of E-cadherin in nCLU-overexpressing PC3 cells is reported in Fig. 5B (nCLU, lanes 1 and 2). Fig. 5B shows transfected PC3 cells in which: i) nCLU is present both in the cytoplasm and in the nucleus (lane 1); 

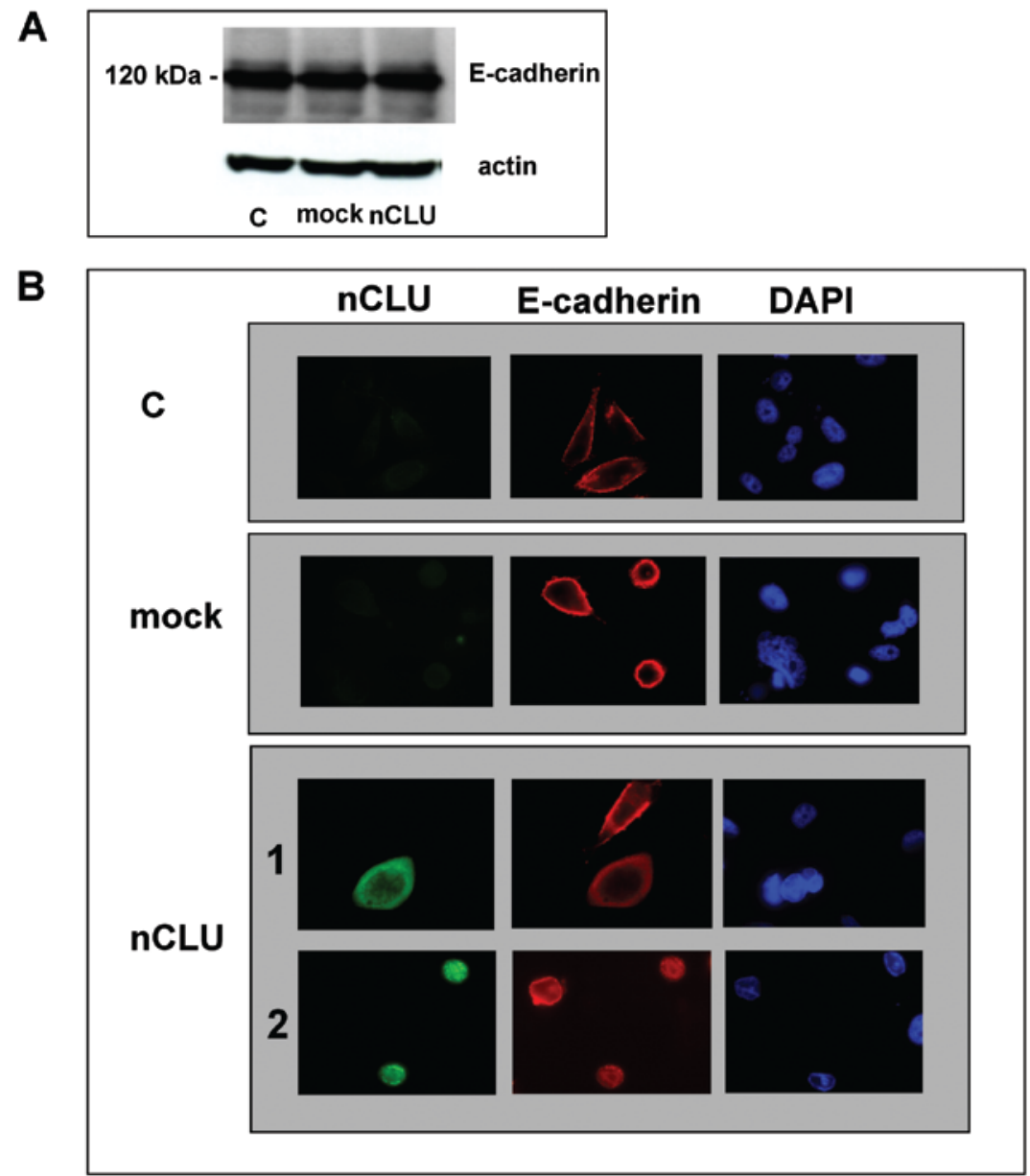

Figure 5. nCLU affects intracellular E-cadherin localization in transfected PC3 prostate cancer cells. (A) Western blot analysis of E-cadherin in whole-cell extracts of transiently $(48 \mathrm{~h})$ transfected PC3 cells. Actin expression is used as a loading control. Representative picture from three experiments done independently, which gave the same results. (B) Immunofluorescence staining of E-cadherin in untransfected control (C), mock-transfected (mock), and nCLU-transfected (nCLU) PC3 cells, $48 \mathrm{~h}$ after transfection. Lane 1, a trasfected PC3 cell in which nCLU is present both in the cytoplasm and in the nucleus; lane 2, a PC3 transfected cell in which most nCLU has entered the nucleus. In both cases, E-cadherin staining at the cell surface disappears and the adhesion molecule appears to be diffusely distributed into the cell. Images were taken at magnification x630.

ii) most of nCLU has already entered the nucleus (lane 2). In both cases, E-cadherin staining at the cell surface disappears and the adhesion molecule appears to be diffusely distributed into the cell, suggesting a complete loss of cell-cell contacts. Interestingly, lanes 1 and 2 show that, in cells in which nCLU could not be efficiently transfected, E-cadherin staining is mainly localized at the cell periphery, as observed in untransfected controls and in mock-transfected controls (Fig. 5B, C and mock).

$n C L U$ reduces prostate cancer cell invasion and MMP-2 activity. A major hallmark of metastasis development is the invasion of tumor cells into surrounding tissues. Metalloproteinases, secreted by tumor cells, play a crucial role in this process. We investigated the effects of nCLU overexpression on the invasive ability of PC3 cells, by using invasion chambers with ECM-Matrigel-coated membranes. The invasion rate of nCLU overexpressing cells was significantly lower than that of either control or mock-transfected cells (Fig. 6A, nCLU vs. $\mathrm{C}$ and mock). Transfection of PC3 cells with nCLU also significantly decreased MMP-2 activity in the culture medium of the cells (Fig. 6B).

\section{Discussion}

We sought to investigate the molecular mechanisms underlying the antitumor activity of the nuclear isoform of clusterin (nCLU) in androgen-independent prostate cancer cells. First, we confirmed our previous observations (40) indicating that nCLU overexpression is associated with a decreased cell motility and a dramatic disassembly of the actin cytoskeleton. We then demonstrated that nCLU significantly decreases the levels of the LIMK1, thus increasing the levels of the unphoshorylated, (active) form of cofilin, the well known actin depolymerizing factor. In a previous report, we showed that nCLU binds to $\alpha$-actinin, an actin cross-linking protein, and hypothesized that this binding would reduce the ability of nCLU to cross-link actin filaments (40). The results reported herein indicate an additional mechanism through which nCLU might cause the disassembly of the actin cytoskeleton, further supporting the dramatic effects of nCLU on prostate cancer cell motility.

In the present study, we also investigated whether nCLU might interact with other components of the cell cytoskeleton, known to be deeply involved in the mechanisms of cancer cell 


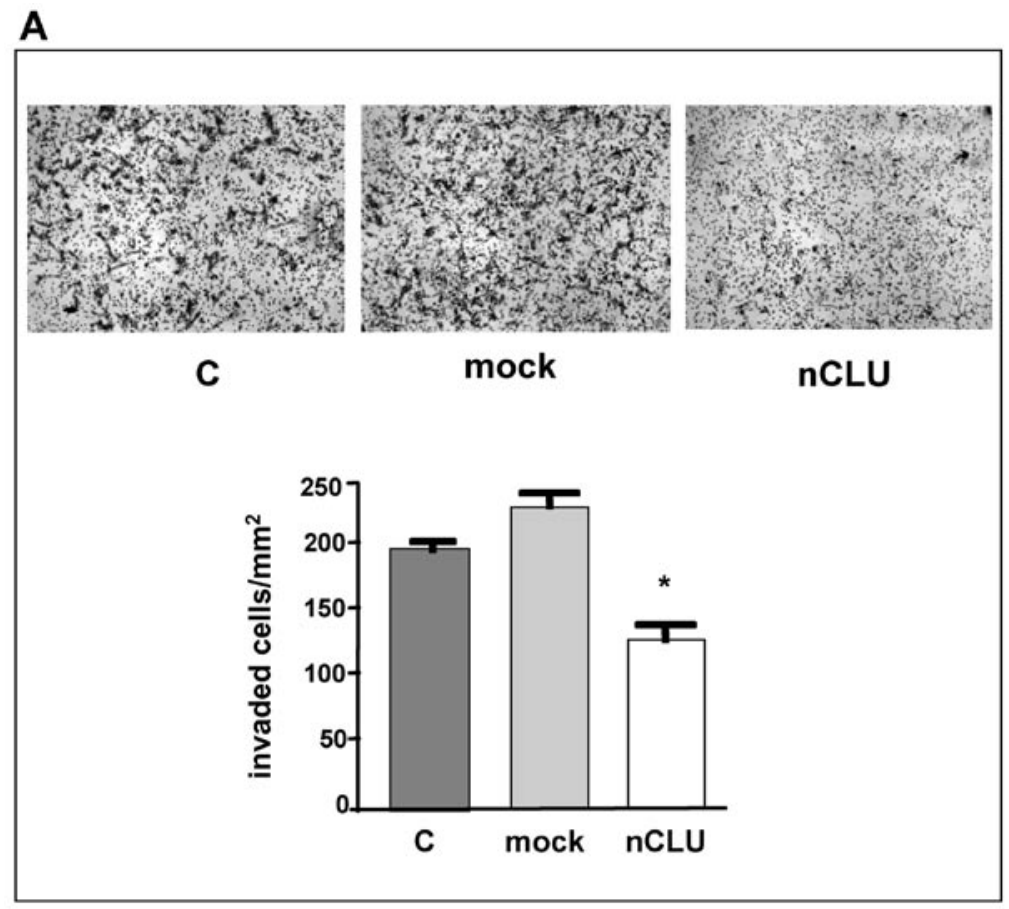

B

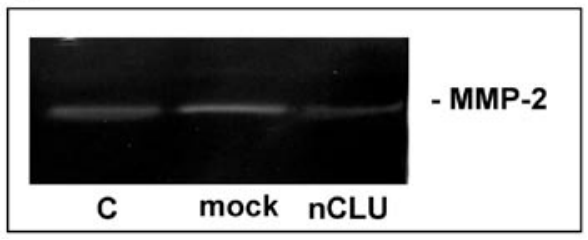

Figure 6. nCLU reduces the invasive behavior of transfected PC3 prostate cancer cells and MMP-2 activity. (A) Untransfected control (C), mock-transfected (mock), and nCLU-transfected (nCLU) PC3 cells were analyzed (48 h after transfection) in an invasion assay, performed using invasion chambers with ECMMatrigel-coated membranes. Shown are representative photographs of membranes depicting the invading cells (upper panel). The data were then quantified (lower panel). ${ }^{*} \mathrm{P}<0.05$ vs. C and mock. (B) Zymographic analysis of the MMP-2 gelatinolytic activity in the culture medium of control (C), mock-transfected (mock), and nCLU-transfected (nCLU) PC3 cells, $48 \mathrm{~h}$ after transfection. Representative pictures from three experiments done independently, which gave the same results.

motility, such as the intermediate filament protein vimentin (46). We found that, in control PC3 cells, vimentin is mainly localized at the cell periphery, accumulated at adhesion sites, as previously described (47). After nCLU overexpression, the distribution of the cytoskeletal protein is completely different, with vimentin being diffusely distributed into the cell. Moreover, we demonstrated that nCLU specifically binds to, and colocalize with vimentin; this suggests that the nuclear clusterin isoform, by sequestering vimentin, may interfere with its crucial roles in the control of cell motility and invasion. These data further support the chaperone activity of clusterin.

The well-known cell adhesion molecule E-cadherin is strictly associated with both actin and vimentin in regulating cell motility and invasive processes. We observed that, in untransfected control PC3 cells, E-cadherin is expressed at the cell periphery, confirming its engagement in the formation of adherent junctions. On the other hand, the peripheral clusters of E-cadherin disappear and the distribution of the adhesion molecule becomes diffuse when nCLU is transfected in prostate cancer cells. Since the expression of E-cadherin at the protein level is not affected by nCLU, we believe that the change in cell distribution of E-cadherin might be directly linked to the dismantling of the cell cytoskeleton.

Fluorescence analysis of nCLU intracellular localization demonstrate that, after its overexpression, nCLU can be detected both at the cytoplasmic and at the nuclear level, confirming our previous observations (40). Moreover, the demonstration that nCLU remarkably affects the cytoskeleton organization further supports the concept that this clusterin isoform may exert some effects (antimotility) at the cytoplasmic level and other effects (proapoptotic) at the nuclear level. Specifically, nCLU might interfere with the migratory behavior of the cell before entering the nucleus; alternatively, it might behave as a shuttle protein from the cytoplasm to the nucleus and vice versa to exert its specific antitumor effects.

The invasive behavior is another hallmark of highly metastatic tumors, such as androgen-independent prostate cancer. Here, we demonstrated that nCLU significantly reduces the invasive behavior of PC3 cells, as well as the amount of the active metalloproteinase MMP-2 released from these cells. Interestingly, LIMK1 activity has been associated with increased cell invasion and matrix protein degradation (43). Thus, by reducing LIMK1 expression, nCLU might affect 
both cell migration (through the disassembly of the actin cytoskeleton) and cell invasion.

Taken together, the results demonstrate that nCLU is endowed with a potent antimetastatic activity, by reducing both cell motility (through its interaction with the cytoskeletal filaments and cell-adhesion molecules) and invasive behavior (by decreasing metalloproteinase activity).

The so-called epithelial-to-mesenchymal transition (EMT) is a process characterized by protein modification and transcriptional events to extracellular stimuli leading to cellular change. Modifications of cell morphology, of cytoskeletal organization (i.e., vimentin), of adhesion molecules at adherent junctions (i.e., E-cadherin), and of extracellular matrix proteolytic enzymes (i.e., MMP-2) are generally considered specific markers of EMT. Thus, the EMT process is typical of cancer cells progressive towards their most aggressive phenotype, characterized by high migratory and invesive behavior. On the basis of these observations, reversal of EMT (mesenchymalepithelial-transition, MET) is now considered a novel and promising strategy to fight cancer. Here, we showed that nCLU reduces the metastatic phenotype of PC3 cells. However, the morphological changes observed in nCLU over-expressing cells do not correspond to those expected for the EMT process; moreover, while vimentin and MMP-2 levels decrease, E-cadherin levels are not affected by nCLU. Thus, our data suggest that the antimetastatic effects of nCLU reported are not correlated with a MET process. It is important to underline that, on the basis of the data so far available in the literature, it is still unclear whether PC3 cells might represent a typical model of the EMT process in prostate cancer (50). PC3 cells have been shown either to possess epithelial features, and to change from the epithelial to a mesenchymal phenotype when challenged with different growth factors (EGF, PDGF, TGF) (51-53) or to display mesenchymal characteristics and to undergo a mesenchymalepithelial transition in other experimental conditions, such as blocking of the Wnt signaling (54). These observations are in line with the results herein reported, demonstrating that the epithelial marker E-cadherin is expressed in most, but not in all, PC3 cells (both controls and mock-transfected). At present, we believe that further characterization of androgenindependent prostate cancer cell lines and signaling pathways is urgently needed to identify an accurate model of the EMT process in prostate cancer.

The proapoptotic activity of nCLU has been previously reported. In prostate cancer cells, nCLU has been consistently shown to accumulate at the nuclear level in cells with different proapoptotic signals $(26,30,38,39,55)$. Moreover, using an experimental system in which a truncated nCLU form $(49 \mathrm{kDa})$ was produced in PC3 cells, we showed that nuclear localization of clusterin significantly reduced the growth and motility of metastatic PC3 prostate cancer cells (40). The data reported herein further support the anti-tumor activity of nuclear clusterin.

The data so far available indicate that the sCLU and nCLU differentially affect prostate tumor growth and progression. It is now generally accepted that a shifting balance between the two clusterin isoforms occurs during prostate epithelial cells transformation, with the prosurvival sCLU prevailing over the proapoptotic/anti-metastatic nCLU $(26,40,56)$. Our data further support the concept that lowering the sCLU/nCLU ratio might represent a successful strategy to control prostate cancer progression. Antisense oligonucleotides targeting sCLU expression have been showed to induce apoptosis and to reduce chemoresistance in $\mathrm{PCa}$ cells in prostate cancer patients $(57,58)$. Novel strategies aimed at specifically increasing nCLU expression/activity might represent a new anti-cancer therapy.

\section{References}

1. Jemal A, Siegel R, Ward E, Hao Y, Xu J and Thun MJ: Cancer statistics 2009. CA Cancer J Clin 59: 225-249, 2009.

2. Javidan J, Deitch AD, Shi XB and De Vere White RW: The androgen receptor and mechanisms for androgen independence in prostate cancer. Cancer Invest 23: 520-528, 2005.

3. Lee ECY and Tenniswood MPR: Emergence of metastatic hormone-refractory disease in prostate cancer after anti-androgen therapy. J Cell Biochem 91: 662-670, 2004.

4. Garmey EG, Sartor O, Halabi S and Vogelzang NJ: Second-line chemotherapy for advanced hormone-refractory prostate cancer. Clin Adv Hematol Oncol 6: 118-122, 2008.

5. Di Lorenzo G, Buonerba C, Autorino R, De Placido S and Sternberg CN: Castration-resistant prostate cancer. Current and emerging treatment strategies. Drugs 70: 983-1000, 2010.

6. Harzstark AL and Small EJ: Castrate-resistant prostate cancer: therapeutic strategies. Expert Opin Pharmacother 11: 937-945, 2010.

7. Jones SE and Jomary C: Clusterin. Int J Biochem Cell Biol 34: 427-431, 2002.

8. Shannan B, Seifert M, Leskov K, Willis J, Boothman D, Tilgen W and Reichrath J: Challenge and promise: roles for clusterin in pathogenesis, progression and therapy of cancer. Cell Death Differ 13: 12-19, 2006.

9. Purrello M, Bettuzzi S, Di Pietro C, Mirabile E, Di Blasi M, Rimini R, Grzeschik KH, Ingletti C, Corti A and Sichel G: The gene for SP-40,40, human homolog of rat sulphated glycoprotein 2 , rat clusterin, and testosterone-repressed prostate message 2, maps to chromosome 8. Genomics 10: 151-156, 1991.

10. Kirszbaum L, Bozas SE and Walker ID: SP-40,40, a protein involved in the control of the complement pathway, possesses a unique array of disulphide bridges. FEBS Lett 297: 70-76, 1992.

11. Rizzi F, Coletta M and Bettuzzi S: Clusterin (CLU): from one gene and two transcript to many proteins. Adv Cancer Res 104: 9-23, 2009.

12. Yang CR, Leskov K, Hosley-Eberlein K, Criswell T, Pink JJ, Kinsella TJ and Boothman DA: Nuclear clusterin/XIP8, and $\mathrm{X}$-ray-induced Ku70-binding protein that signals cell death. Proc Natl Acad Sci USA 97: 5907-5912, 2000.

13. Scaltriti M, Santamaria A, Paolucci R and Bettuzzi S: Intracellular clusterin induces G2-M phase arrest and cell death in PC-3 prostate cancer cells. Cancer Res 64: 6174-6182, 2004.

14. Reddy KB, Jin G, Karode MC, Harmony JA and Howe PH: Transforming ghrowth factor $\beta$ (TGF $\beta$ )-induced nuclear localization of apolipoprotein $\mathrm{J} /$ clusterin in epithelial cells. Biochemistry 35 : 6157-6163, 1996.

15. Leskov K, Klokov D, Li J, Kinsella TJ and Boothman DA: Synthesis and functional analyses of nuclear clusterin: a cell death protein. J Biol Chem 278: 11590-11600, 2003.

16. Redondo M, Villar E, Torres-Munoz J, Tellez T, Morell M and Petito CK: Overexpression of clusterin in human breast carcinoma. Am J Pathol 157: 393-399, 2000.

17. Pucci S, Bonanno E, Pichiorri F, Angeloni C and Spagnoli LG: Modulation of different clusterin isoforms in human colon tumorigenesis. Oncogene 23: 2298-2304, 2004.

18. Yang G, Li XM and Xie D: Overexpression of clusterin in ovarian cancer is correlated with impaired survival. Int $\mathbf{J}$ Gynecol Cancer 19: 1342-1346, 2009.

19. Bi J, Guo AL, Lai YR, Li B, Zhong JM, Wu HQ, Xie Z, He YL, Lv ZL, Lau SH, Wang Q, Huang XH, Zhang LJ, Wen JM and Guan XY: Overexpression of clusterin correlates with tumor progression, metastasis in gastric cancer: a study on tissue microarray. Neoplasma 57: 191-197, 2010.

20. Mazzarelli P, Pucci S and Spagnoli LG: CLU and colon cancer. The dual face of CLU: from normal to malignant phenotype. Adv Cancer Res 105: 45-61, 2009. 
21. Park DC, Yeo SG, Wilson MR, Yerbury JJ, Kwong J, Welch WR, Choi YK, Birrer MJ, Mok SC and Wong KK: Clusterin interacts with Paclitaxel and confers Paclitaxel resistance in ovarian cancer. Neoplasia 10: 964-972, 2008.

22. Djeu JY and Wei S: Clusterin and chemoresistance. Adv Cancer Res 105: 77-92, 2009.

23. Wei L, Xue T, Wang J, Chen B, Lei Y, Huang Y and Xin X: Roles of clusterin in progression, chemoresistance and metastasis of human cancer. Int J Cancer 125: 791-806, 2009.

24. Watari H, Kanuma T, Ohta Y, Hassan M, Mitamura T, Hosaka M, Minegishi T and Sakuragi N: Clusterin expression inversely correlates with chemosensitivity and predicts poor survival in patients with locally advanced cervical cancer treated with cisplatin-based neoadjuvant chemotherapy and radical hysterectomy. Pathol Oncol Res 16: 345-352, 2010.

25. Dia VP and Mejia EG: Lunasin promotes apoptosis in human colon cancer cells by mitochondrial pathway activation and induction of nuclear clusterin expression. Cancer Lett 295: 44-53, 2010.

26. Rizzi $\mathrm{F}$ and Bettuzzi S: The clusterin paradigm in prostate and breast carcinogenesis. Endocr Relat Cancer 17: R1-R17, 2010.

27. Steinberg J, Oyasu R, Lang S, Sintich S, Rademaker A, Lee C, Kozlowski JM and Sensibar JA: Intracellular levels of SGP-2 (Clusterin) correlates with tumor grade in prostate cancer. Clin Cancer Res 3: 1707-1711, 1997.

28. Scaltriti M, Brausi M, Amorosi A, Caporali A, D'Arca D, Astancolle S, Corti A and Bettuzzi S: Clusterin (SGP-2, ApoJ) expression is downregulated in low- and high-grade human prostate cancer. Int J Cancer 108: 23-30, 2004.

29. Rizzi F, Belloni L, Crafa P, Lazzaretti M, Remondini D, Ferretti S, Cortellini P, Corti A and Bettuzzi S: A novel gene signature for molecular diagnosis of human prostate cancer by RT-qPCR PLoS ONE 3: e3617, 2008.

30. Rizzi F and Bettuzzi S: Clusterin (CLU) and prostate cancer. Adv Cancer Res 105: 1-19, 2009.

31. Girard FP, Byme J, Downes M, Fanning D, Desgrandchamps F, Fitzpatrick JM and Watson RW: Detecting soluble Clusterin in in vitro and in vivo models of prostate cancer. Neoplasma 57: 488-493, 2010

32. Miyake H, Muramaki M, Furukawa J, Kurahashi T and Fujisawa M: Serum level of clusterin and its density in men with prostate cancer as novel biomarkers reflecting disease extension. Urology 75: 454-459, 2010.

33. Miyake H, Nelson C, Rennie PS and Gleave ME: Acquisition of chemoresistant phenotype by overexpression of the antiapoptotic gene testosterone-repressed prostate message- 2 in prostate cancer xenograft models. Cancer Res 60: 2547-2554, 2000.

34. Zhang H, Kim JK, Edwards CA, Xu Z, Taichman R and Wang CY: Clusterin inhibits apoptosis by interacting with activated bax. Nat Cell Biol 7: 909-915, 2005.

35. Sallman DA, Chen X, Zhong B, Gilvary D, Zhou J, Wei S and Djeu JY: Clusterin mediates TRAIL resistance in prostate tumor cells. Mol Cancer Ther 6: 2938-2947, 2007.

36. Ammar $\mathrm{H}$ and Closset JL: Clusterin activates survival through the phosphatidylinositol 3-kinase/Akt pathway. J Biol Chem 283: 12851-12861, 2008.

37. Zhong B, Sallman DA, Gilvary DL, Pernazza D, Sahakian E, Fritz D, Cheng JQ, Trougakos I, Wei S and Djeu JY: Induction of clusterin by AKT-role in cytoprotection against docetaxel in prostate tumor cells. Mol Cancer Ther 9: 1831-1841, 2010

38. Scaltriti M, Bettuzzi S, Sharrard RM, Caporali A, Caccamo AE and Maitland NJ: Clusterin overexpression in both malignant and nonmalignant prostate epithelial cells induces cell cycle arrest and apoptosis. Br J Cancer 91: 1842-1850, 2004.

39. Caccamo AE, Scaltriti M, Caporali A, D'Arca D, Scorcioni F, Candiano G, Mangiola M and Bettuzzi S: Nuclear translocation of a clusterin isoform is associated with induction of anoikis in SV40-immortalized human prostate epithelial cells. Ann NY Acad Sci 1010: S514-S519, 2003.
40. Moretti RM, Montagnani Marelli M, Mai S, Cariboni A, Scaltriti M, Bettuzzi S and Limonta P: Clusterin isoforms differentially affect growth and motility of prostate cells: possible implications in prostate tumorigenesis. Cancer Res 67 10325-10333, 2007.

41. Caccamo AE, Scaltriti M, Caporali A, D'Arca D, Corti A, Corvetta D, Sala A and Bettuzzi S: $\mathrm{Ca}^{2+}$ depletion induces nuclear clusterin, a novel effector of apoptosis in immortalized human prostate cells. Cell Death Differ 12: 101-104, 2005.

42. Lee SH and Dominguez R: Regulation of actin cytoskeleton dynamics in cells. Mol Cells 29: 311-325, 2010.

43. Scott RW and Olson MF: LIM kinases: function, regulation and association with human disease. J Mol Med 85: 555-568, 2007.

44. Jordan MA and Wilson L: Microtubules as a target for anticancer durgs. Nat Rev Cancer 4: 253-265, 2004.

45. Etienne-Manneville S: Actin and microtubules in cell motility: which one is in control? Traffic 5: 470-477, 2004.

46. Eriksson JE, Dechat T, Grin B, Helfand B, Mendez M, Pallari H-M and Goldman RD: Introducing intermediate filaments: from discovery to disease. J Clin Invest 119: 1763-1771, 2009.

47. Lang SH, Hyde C, Reid IN, Hitchcock IS, Hart CA, Bryden AAG, Villette J-M, Stower MJ and Maitland NJ: Enhanced expression of vimentin in motile prostate cell lines and in poorly differentiated and metastatic prostate carcinoma. Prostate 52: 253-263, 2002.

48. Zhao Y, Yan Q, Long X, Chen X and Wang Y: Vimentin affects the mobility and invasiveness of prostate cancer cells. Cell Biochem Funct 26: 571-577, 2008.

49. Yilmaz $M$ and Christofori G: Mechanisms of motility in metastasizing cells. Mol Cancer Res 8: 629-642, 2010.

50. Hugo H, Ackland ML, Blick T, Lawrence MG, Clemens JA, Williams ED and Thompson EW: Epithelial-mesenchymal and mesenchymal-epithelial transitions in carcinoma progression. J Cell Physiol 213: 374-383, 2007.

51. Kong D, Li Y, Wang Z, Banerjee S, Ahmad A, Kim HR and Sarkar FH: miR-200 regulates PDGF-D-mediated epithelialmesencymal transition, adhesion, and invesion of prostate cancer cells. Stem Cells 27: 1712-1721, 2009.

52. Gan Y, Shi C, Inge L, Hibner M, Balducci J and Huang Y: Differential roles of ERK and Akt pathways in regulation of EGFR-mediated signaling and motility in prostate cancer cells. Oncogene 29: 4947-4958, 2010.

53. Lenferink AE, Cantin C, Nantel A, Wang E, Durocher $Y$, Banville M, Paul-Roc B, Marcil A, Wilson MR and O'ConnorMcCourt MD: Transcriptome profiling of TGF- $\beta$-induced epithelial-to-mesenchymal transition reveals extracellular clusterin as a target for therapeutic antibodies. Oncogene 29: 831-844, 2010.

54. Yee DS, Tang Y, Liu Z, Guo Y, Ghaffar S, McQueen P, Atreya D, Xie J, Simoneau AR, Hoang BH and Zi X: The Wnt inhibitory factor 1 restoration in prostate cancer cells was associated with reduced tumor growth, decreased capacity of cell migration and invasion and a reversal of epithelial to mesenchymal transition. Mol Cancer 9: 162-175, 2010.

55. Bettuzzi S and Rizzi F: Nuclear CLU (nCLU) and the fate of the cell. Adv Cancer Res 104: 59-88, 2009.

56. Pucci S and Bettuzzi S: The shifting balance between CLU forms during tumor progression. Adv Cancer Res 104: 25-32, 2009.

57. Miyake H, Hara I, Fujisawa M and Gleave ME: The potential of clusterin inhibiting antisense oligodeoxynucleotide therapy for prostate cancer. Expert Opin Investig Drugs 15: 507-517, 2006.

58. Di Cresce $\mathrm{C}$ and Koropatnick J: Antisense treatment in human prostate cancer and melanoma. Curr Cancer Drug Targets 10: $555-565,2010$. 